\title{
Detecting Atmospheric Rivers in Large Climate Datasets
}

\author{
Surendra Byna \\ Lawrence Berkeley Laboratory \\ 1 Cyclotron Road, 50B-3238 \\ Berkeley, CA 94720 \\ USA \\ SByna@lbl.gov
}

\author{
Prabhat \\ Lawrence Berkeley Laboratory \\ 1 Cyclotron Road, 50F-1650 \\ Berkeley, CA 94720 \\ USA \\ prabhat@hpcrd.lbl.gov \\ Kesheng Wu \\ Lawrence Berkeley Laboratory \\ 1 Cyclotron Road, 50B-3238 \\ Berkeley, CA 94720 \\ USA \\ KWu@lbl.gov
}

Michael F. Wehner

Lawrence Berkeley Laboratory

1 Cyclotron Road, 50F-1650

Berkeley, CA 94720

USA

MFwehner@lbl.gov

\begin{abstract}
Extreme precipitation events on the western coast of North America are often traced to an unusual weather phenomenon known as atmospheric rivers. Although these storms may provide a significant fraction of the total water to the highly managed western US hydrological system, the resulting intense weather poses severe risks to the human and natural infrastructure through severe flooding and wind damage. To aid the understanding of this phenomenon, we have developed an efficient detection algorithm suitable for analyzing large amounts of data. In addition to detecting actual events in the recent observed historical record, this detection algorithm can be applied to global climate model output providing a new model validation methodology. Comparing the statistical behavior of simulated atmospheric river events in models to observations will enhance confidence in projections of future extreme storms. Our detection algorithm is based on a thresholding condition on the total column integrated water vapor established by Ralph et al. (2004) followed by a connected component labeling procedure to group the mesh points into connected regions in space. We develop an efficient parallel implementation of the algorithm and demonstrate good weak and strong scaling. We process a 30 -year simulation output on 10,000 cores in under 3 seconds.
\end{abstract}

\section{Categories and Subject Descriptors}

J.2 [Computer Applications]: Physical Sciences and Engineering; I.5.4 [Pattern Recognition]: Applications; I.6.6 [Simulation and Modeling]: Simulation Output Analysis

Copyright 2011 Association for Computing Machinery. ACM acknowledges that this contribution was authored or co-authored by an employee, contractor or affiliate of the U.S. Government. As such, the Government retains a nonexclusive, royalty-free right to publish or reproduce this article, or to allow others to do so, for Government purposes only.

PDACO'11, November 14, 2011, Seattle, Washington, USA.

Copyright 2011 ACM 978-1-4503-1130-4/11/11...\$10.00.

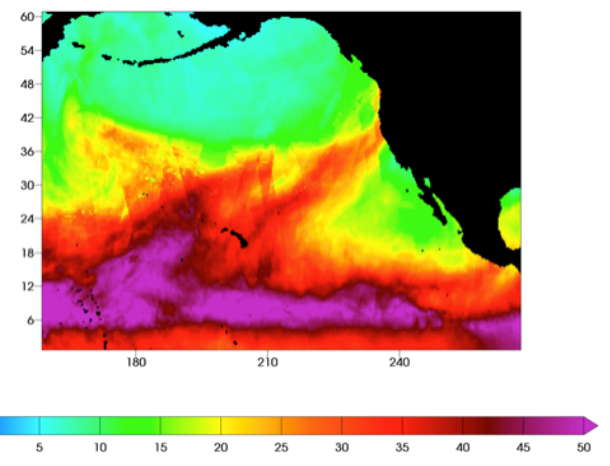

Figure 1: The observed three-day average total precipitable water $(\mathrm{mm})$ on December 14, 2010 from an analysis of SSM/I satellite data (www.rss.com) showing an atmospheric river reaching west cost of US.

\section{General Terms}

Algorithms, Performance, Experimentation

\section{Keywords}

Atmospheric rivers, connected component labeling, extreme climate events, automatic detection of atmospheric rivers

\section{INTRODUCTION}

Extreme precipitation events on the western coast of North America are often traced to an unusual weather phenomenon known as atmospheric rivers (ARs). These events refer to filamentary structures in atmosphere that transport significant amounts of water over a long distance in narrow bands [1][10]. In one of the earliest studies on this phenomenon, it was determined that such a structure could carry more water than the great river Amazon [9]. Figure 1 shows an example of an atmospheric river that deposited record amounts of rainfall on California over the course of several days in December 2010. For regions such as the west coast of the United States, atmospheric rivers bring more than half of the annual total precipitation and 
can occur in as few as five days [1]. Their intensity creates a possibility of flooding and wind damage, yet at the same time they provide a significant amount of the fresh water needed for the western states' water management systems. Although current research is focused on AR events making landfall on the western coast of North America, the phenomena is not limited to the northeastern Pacific and can occur in other ocean basins.

This study of atmospheric rivers is part of on-going efforts to understand the mechanisms responsible for severe but infrequent weather events. In some winter-time events, such as the atmospheric rivers, several planetary-scale conditions must be in phase for such large entrainments of tropical moisture [10]. To reach general conclusions, many such events must be analyzed individually and as a whole set. To analyze these events, they must first be identified.

In this work, we develop an efficient algorithm for identifying the atmospheric rivers from both observational data from satellite measurements and climate model output data. Part of our motivations is to understand the statistical behavior of the events to analyze how they might change in a warmer climate. Hence, a key objective is to develop an efficient algorithm to identify AR from large volumes of data, allowing us to determine the frequency and intensity of AR events. Additional information about the structure of AR events, notably landfall location, intensity and duration are also obtainable by our method and will prove useful in projection of future climate change.

Observed precipitation and offshore wind speed [8] have been used to identify an AR in the western Pacific basin by constructing a scatterplot of high quality hourly precipitation and wind data collected at key coastal weather stations [14]. This ad hoc method is based on setting thresholds of precipitation and wind speed in the upslope direction and has proved useful in identifying recent atmospheric river events. However, this detection method is localized by definition and requires ancillary data, such as total precipitable water from satellite measurements, to characterize the atmospheric river event. Furthermore, as atmospheric rivers can happen in any ocean basin, the scheme would fail if the event does not make landfall where quality observations are available. This likely precludes analyses in a global context. Application to climate simulations may also pose problems due to model bias in precipitation and wind fields. For example, the thresholds appropriate to observations may not work well if model extreme precipitation and winds exhibit systematic errors. In this paper, we present an alternative detection scheme based on examining basin-wide data characteristic of the atmospheric river phenomenon. Our methodology allows for detection and characterization of such events in both satellite measurements and climate model output. As such, it will prove a critical tool in the projection of future changes in this class of extreme storm. The key contributions of this work are as follows:
- We designed an efficient algorithm for identifying AR using total column integrated precipitable water vapor data from either observations or simulations.

- Our algorithm is highly parallelizable; we demonstrate efficient parallel scaling on a large 1TB dataset.

- We verify the results from our algorithm against published studies by using a set of satellite data that have not been previously used for this purpose. The data used in this study is from Advanced Microwave Scanning Radiometer (AMSR-E) satellite described in Section 4 . We obtain classification accuracy of $92 \%$.

\section{Related Work}

In this section, we briefly review related work on atmospheric rivers and feature detection algorithms.

\subsection{Atmospheric Rivers}

An atmospheric river is a long and narrow structure in atmosphere that transports tropical moisture to the far-flung regions outside of the tropical zone [1][10]. Zhu and Newell were the first to name this phenomenon "atmospheric river" noting that they typically transport more water than the Amazon [16]. As they can be highly localized, "river" is an apt description of such a narrow stream of moisture moving at high speeds across thousands of kilometers. AR events occur in oceans around the globe, including the Atlantic basin affecting the British Isles ${ }^{1}$.

The key characteristic recognized in earlier studies of ARs is the moisture flux [17]. However, that quantity turns out to be a hard to directly observe. In 2004, Ralph et al. [11] established a much simpler set of conditions for identify atmospheric rivers in satellite observations. Their detection works with two-dimensional data over a uniform mesh on the global and is primarily based on the Integrated Water Vapor (IWV) content, which measures the total water content (measured in volume) in the volume of atmosphere above a unit of earth surface. This quantity is measured in millimeters $(\mathrm{mm})$ or centimeters $(\mathrm{cm})$. More specifically, they identify atmospheric rivers as atmospheric features with IWV $>2 \mathrm{~cm}$, more than $2000 \mathrm{~km}$ in length and less than $1000 \mathrm{~km}$ in width. Based on this definition, Ralph and colleagues have identified hundreds of atmospheric river events in the data produced by Special Sensor Microwave Imager (SSM/I) satellite observations [1][7].

In this work, we will use a different set of satellite data as well as output data from a state of the art high-resolution climate model. The observational data we use is from a satellite called Advanced Microwave Scanning Radiometer (AMSR-R). This device measures IWV allowing us to use the same conditions as proposed by Ralph et al. [11].

Objective identification of atmospheric river events is a challenging task. Identifying observed events in the historical record for case study analyses can exploit

\footnotetext{
${ }^{1}$ http://cimss.ssec.wisc.edu/goes/blog/archives/3838
} 
associated information such as on-shore extreme precipitation and wind direction to identify candidate events. Large-scale structural information can then be gained by analyses of satellite measurements [10]. However, analyses of the statistical behavior of atmospheric rivers are also necessary to understand the more general relationship to large-scale climatic variations. The ability of climate models to simulate atmospheric river statistics is key to projecting if these phenomena change as the climate warms. Hence, an atmospheric river identification scheme that neither misidentifies nor misses candidate events is critical to the statistical analysis of climate models, and their comparison to the observed recent past.

\subsection{Feature Detection on Mesh Data}

Climate Model and satellite output are typically generated (or regrided) on a regular mesh over the globe. Following the methodology used by Ralph et al., we perform our detection on 2-D data on the latitude-longitude mesh [11]. An atmospheric river is an event that can last for a few days. Our detection algorithm processes one day at a time. For each day's data, the AR appears as a connected region in space where the integrated water vapor content is high. This type of feature in space is commonly known as region of interest. Identifying such regions of interest is a basic operation in many computer visual analysis tasks.

Our detection algorithm proceeds in three steps. The first step performs a thresholding operation based on IWV value; mesh points with high IWV values are marked for further processing. The second step connects the marked mesh points into regions. This step employs a connected component labeling algorithm. The connected regions are passed to the last step for verification of sizes. The first and last steps are relatively straightforward. In this section, we briefly review the algorithms used for connected component labeling [3][15].

The IWV data processed by our feature identification procedure is stored as a 2-dimensional array. The output from the thresholding step can be treated as a binary image, where the foreground pixels are mesh points with large IWV values and the background pixels are mesh points with small IWV values. This allows us to use the connected component labeling algorithms developed from image processing. There are a variety of algorithms for this task. For example, there are a number of different parallel approaches [5][12], some methods using specialized hardware [4][6]. Since the image sizes are relatively modest in our application, we choose to perform connected component labeling using only a single CPU core.

To find the connected component labels, we use a two-pass algorithm that gathers the connectivity information among the foreground pixels and then assign the final labels to each pixel. The two-pass algorithms avoid scanning the image multiple times by manipulating the label equivalence information to arrive at a final assignment for each provisional label. The most efficient data structure for keeping track of the label equivalence information is called union-find [2], and the most efficient implementation of the union-find data structure is an implicit data structure that uses a single array [15]. An efficient union-find implementation is critical to the overall effectiveness of the two-pass algorithm. To keep the computational complexity low, we chose to keep the binary image in a 2-D array.

\section{OUR APPROACH}

Our algorithm processes 2-D meshes defined over the globe. These meshes are relatively small, for example, the satellite observation data is defined on a $1 / 4^{\circ}$ mesh with just over $1 \mathrm{M}$ mesh points, and the climate model output uses a $1 / 2^{\circ}$ mesh. Even with fine meshes at $1 / 10^{\circ}$ mesh, the data associated with a single variable, i.e. integrated water vapor (IWV), can easily fit into main memory. While we need to process many time steps in the complete dataset, this can be done in parallel.

A schematic illustration of the parallel algorithm for AR detection is shown in Figure 2. We divided the algorithm into an I/O phase and a compute phase. The I/O phase includes reading the input filenames and vapor data. The computation phase consists of thresholding, connected component labeling, and verification steps. Each process generates an output indicating the presence or absence of an AR. Our design allows each process to run independently without any need for inter-process synchronization or communication.

\subsection{I/O Phase}

Our current implementation requires a list of data file names to process. This list is currently stored in a single, shared file. Currently, all processes read the file; it is possible to split this file in the future to reduce metadata overhead. Once each process determines what file to process, it then proceeds with reading the IWV data.

The function that performs the reading of IWV data takes a number of optional input parameters, such as granularity of climate data, type of data format (such as gunzip compressed format, netCDF, etc.), the number of time steps present in one day's data and regions where AR should be

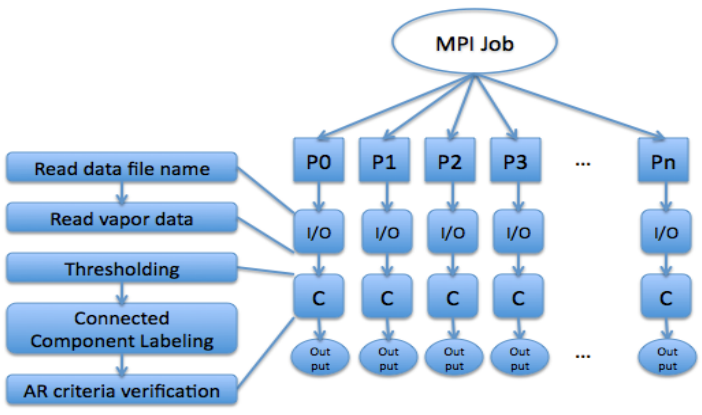

Figure 2: AR detection tool implemented with MPI 
detected. This flexibility allows us to detect AR in any region of the world at different granularity.

\subsection{Compute Phase}

\subsubsection{Thresholding}

In the compute phase, the first step is a set of thresholding operations on IWV values. Ralph et al. [11] specify IWV values $>20 \mathrm{~mm}$ for detecting atmospheric rivers. We use this threshold value for all results reported in the paper. However, our detection tool can take on a pair of thresholds that define the lower bound and an upper bound for the IWV values. This additional flexibility can be useful for with systematic biases. The output of the thresholding step is a collection of mesh points that satisfy the threshold criteria. These "foreground" pixels are then processed by the Connected Component Labeling (CCL) step.

\subsubsection{Connected Component Labeling}

Our connected component labeling implementation is based on a two-pass algorithm [15]. The algorithm can be broken down into three steps. The first step assigns a provisional label to each mesh point visited. These provisional labels may turn out to be assigned to connected mesh points. We say that these labels are equivalent. This label equivalence information is recorded in a data structure called union-find. The second step works with the union-find data structure to determine the final label for each provision label. The third step replaces the provisional labels with their final values. This third step is a series of straightforward assignments.

The first step examines each mesh point in turn. A mesh point failing the thresholding conditions will receive a special label, say 0 , to indicate that it is not of interest. A mesh point satisfying the thresholding conditions will receive a provisional label. This assignment proceeds as follows. If there is no neighbor with a provisional label already, then this mesh point receives a new label. If any of its neighbors have already received a label, any of their labels can be assigned to the current mesh point. Because the neighbors are connected to this mesh point and to each other, their labels should be the same. We say that these labels are equivalent, and choose the smallest labels as the "representative" of the groups of equivalent labels.

The union-find data structure stores the label equivalence information. This data structure supports two key operations called union and find. Given any provisional label, the find operation locates its "representative". Given any two provisional labels, the union operation is to record that they are equivalent to each other. This operation can be implemented as two find operations followed by an
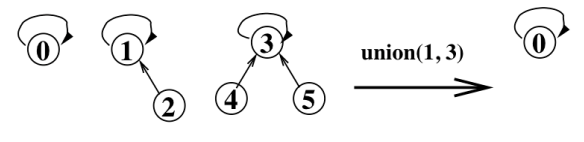

Find $(4)=3$

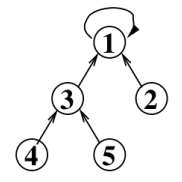

Figure 3: An array representation of the rooted trees operation to set one "representative" pointing to the other. We choose to have the representative with larger numerical value pointing to the representative with smaller value. The union-find data structure can be interpreted as representing a forest of union-find trees, where the "representative" is the root of each tree. Pictorially, this is illustrated in Figure 3. By choosing to use non-negative integers as labels, it is possible to use the labels as the array index and implement the union-find data structure in a single array as illustrated in Figure 3.

Using an array to implicitly represent the union-find trees has the advantage that the memory for the union-find data structure is consecutive in memory. Furthermore, the find operations always traverse to the left in Figure 3. This predictable pattern reduces the average cost of the memory accesses, which improves the overall effectiveness of the labeling algorithm.

\subsubsection{Verification}

After the connected component labeling step, each connected group of mesh points receives a unique label for identification. We then compute the length and width of each group, and impose the relevant constraints (i.e. Length $>2000 \mathrm{~km}$ and Width $<1000 \mathrm{~km}$ [11]) in the verification step. To compute the length, we find the medial axis of a connected component label. Since each pixel on the globe has a relatively constant area, by counting the number of pixels in the connected component label, we compute the area and then the average width by dividing the area with the medial axis length. The detection tool classifies an AR event that satisfies all the criteria.

\section{EXPERIEMENTAL METHODOLOGY}

Thus far, we have described the algorithm for detecting atmospheric rivers. We are interested in evaluating the performance of our algorithm along the following metrics:

- How well does our algorithm perform? What is its accuracy?

- How well does the implementation scale with large data (weak scaling)?

- How well does the implementation scale with number of processes (strong scaling)?

We conducted our experiments on the NERSC Cray XE6 supercomputing system Hopper. The system has $\sim 6,400$ compute nodes, with 24 cores (total $\sim 150,000$ cores, 2 twelve-core AMD 'MagnyCours' $2.1 \mathrm{GHz}$ processors per node) and $32 \mathrm{~GB}$ memory per node. We used all 24 cores of a node for our tests and have one MPI process on each core. Hopper uses Lustre as its file system, with a peak theoretical I/O bandwidth of $35 \mathrm{~GB} / \mathrm{s}$. The Lustre system is configured with 156 Object Storage Targets (OSTs). We now describe our experimental methodology for addressing these questions. 


\subsection{Accuracy of our Approach}

After considering a number of approaches to validate the accuracy of our detection algorithm, we settled on comparing our results to the published AR events in the west coast US by a number of other researchers [1][7]. These papers contain an exhaustive list of atmospheric rivers reaching the US west coast from the year 1998-2008. We treat the results reported in Dettinger et al. [1] from June 2002 and 2008 as ground truth. We note that our results are obtained from a different satellite, Advanced Microwave Scanning Radiometer (AMSR-E) satellite (http://www.ssmi.com/).

\subsection{Weak Scaling}

The field of climate modeling is undergoing active research: we expect larger and larger simulation datasets to be produced in the coming years. While the dataset sizes are increasing, we also have access to large supercomputing systems to process the data. Hence, it is important that data analysis programs are able to scale up as more computing resources are provide for large data sets. To measure this type of scalability, we keep the work given to each process constant, but increasing the number of processes across 1000, 2000, 4000, 8000, and 10000 MPI processes, while proportionally increasing the problem sizes from 50GB to $1 \mathrm{~TB}$. We will report both the time to read the input data and the time to complete the computations. In measuring the $\mathrm{I} / \mathrm{O}$ performance, we will report the I/O throughput instead of the more common read or write speed. There is no synchronization among the processes; therefore, the I/O operations on each process are not coordinated.

\subsection{Strong Scaling}
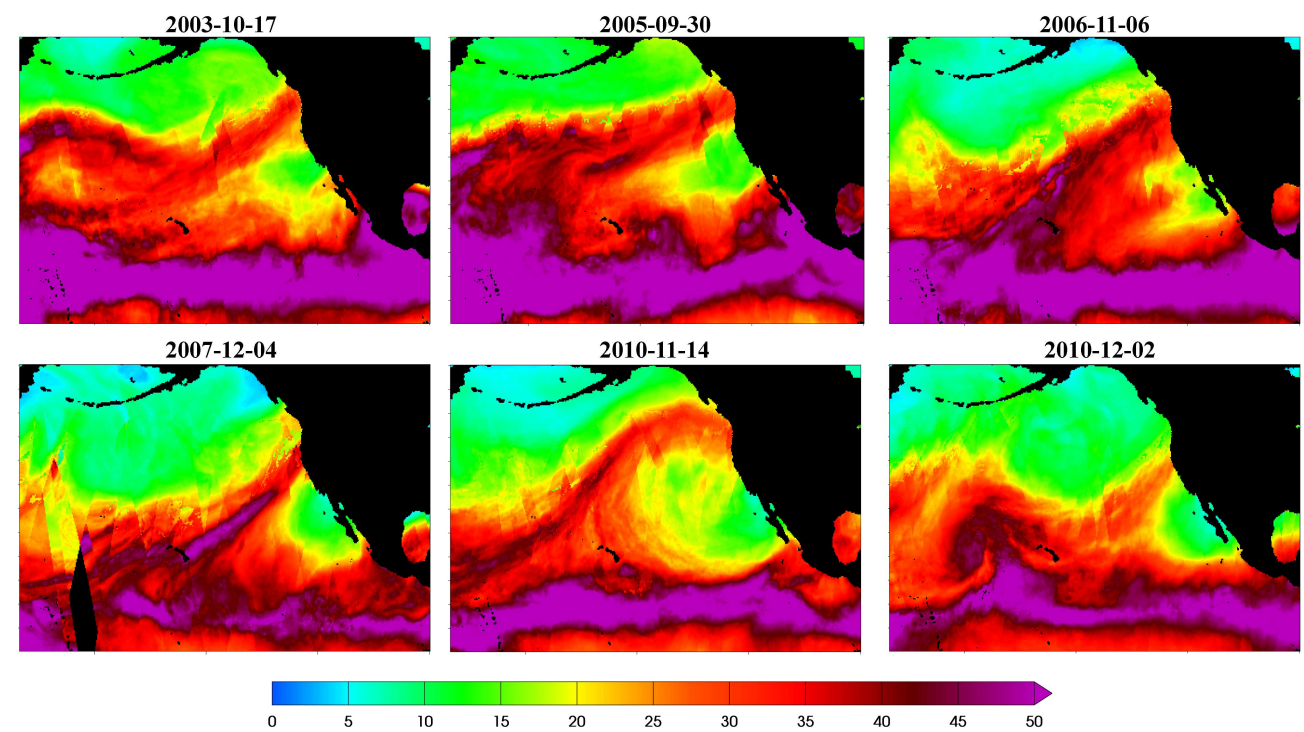

Figure 4: Some typical atmospheric river events detected by our algorithm from the observational dataset. Shown is total column integrated precipitable water in $\mathrm{mm}$. Note that the structure of each event is unique. Also note that data irregularities in the satellite measurements (seen as abrupt discontinuities e.g. in the 2007-12-04 event) do not have an adverse effect on the detection. 


\subsubsection{Model Data}

We use climate data generated by the finite volume version of the Community Atmospheric Model (fvCAM) in our scalability study [13]. The fvCAM uses a finite volume approximation to the atmospheric equations of motion and have been specifically optimized for parallel execution.

Output data in netCDF format includes multiple variables such as pressure, humidity, temperature, total vertically integrated water vapor. For detecting atmospheric rivers, we use the data value for the integrated water vapor. The data is arranged in a $361 \times 576$ mesh, which represents $0.5^{\circ}$ latitude by $0.625^{\circ}$ longitude. There are 4 simulated time steps per one day, i.e. one per every 6 hours, and the total dataset contains 15 simulated years worth of data that amounts nearly $450 \mathrm{~GB}$. The dataset is stored into 1095 files and each file consists of 5 days worth of data.

To avoid dealing with intra-day variations, our detection algorithm works with daily averages calculate from the 6hour time steps within the day. Since model data does not have any missing data, we did not need to compute the average for 3 days as in the observational data. In our strong scaling tests, we used data related to 10,000 days, which is $\sim 1 \mathrm{~TB}$. In the weak scaling experiments, the data size is increased in proportion with the number of processes used. In these, each MPI process analyzes data related to one day. For example, in a 10,000 process MPI job, the application processes 10,000 days worth of data, which is in the range of $1 \mathrm{~TB}$. Similar to the observational data analysis, in both weak scaling and strong scaling studies, we analyzed data related coastal areas of California, Oregon, and Washington states. The tool can be used for detecting AR in any region, by changing the longitude and latitude bounding box parameters and the AR detection criteria.

\section{RESULTS}

We outlined three questions to address in our performance study. In this section, we report our findings on each separately.

\subsection{Classifier Performance}

We applied our AR detection tool to the observational data, and compare the detected events with the published paper by Dettinger et al. [1] We use the same thresholds listed in their paper: water vapor $(>20 \mathrm{~mm})$, length $(>2000 \mathrm{~km})$ and width $(<1000 \mathrm{~km})$, and spatial constraints of examining ARs originating in the tropics and making landfall on the western US coast. Figure 4 shows a sampling of detections from our program.

Our tool detected $81 \%$ of the AR events reported in Dettinger, et al. Upon further examination, we discovered that Dettinger, et al. were reporting ARs that were wider than $1000 \mathrm{~km}$ and the rivers that did not actually make landfall (but was close to it). We thereafter removed entries
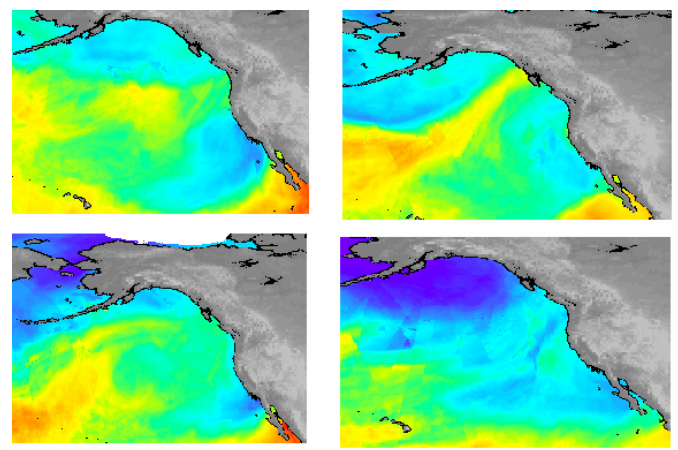

Figure 5: Samples of undetected events by our program

from Dettinger, et al.'s list that did not make landfall. The resulting accuracy of our tool is $92 \%$. Figure 5 shows a sample of "rivers" classified by Dettinger et al. but not detected by our algorithm. Our tool detected only the events that reached the western states of the US as we set the states as the region of interest. These events have vapor below threshold in some parts of the narrow band and some are wider than $1000 \mathrm{~km}$. Since these connected labels do not fit in the source, destination, length, and width criteria, they are not detected as AR by our tool.

Figure 6 shows statistics of AR events between 2002 and 2010. For year 2002, the data is available from June to December, and for all other years, the events are for the whole year. We counted consecutive days with an AR as one event. We separate the AR events in the winter-time from summer months. This relative distribution is quite similar to those reported in earlier studies [1][7].

\subsection{Weak Scaling}

Figure 7 shows results from our weak scaling experiment. The $\mathrm{x}$-axis shows number of MPI processes, and the $\mathrm{y}$-axis shows the time in seconds (in logarithmic scale). To recall the experimental setup, each process analyzes data for a single day; as more processes as added, the detection algorithm works on a proportionally larger number of days.

We observe that majority of the execution time of our tool is dominated by $\mathrm{I} / \mathrm{O}(\sim 98 \%)$. Since each process only works on one day's worth of data, we expect that the I/O time and the computation time to remain constant as the

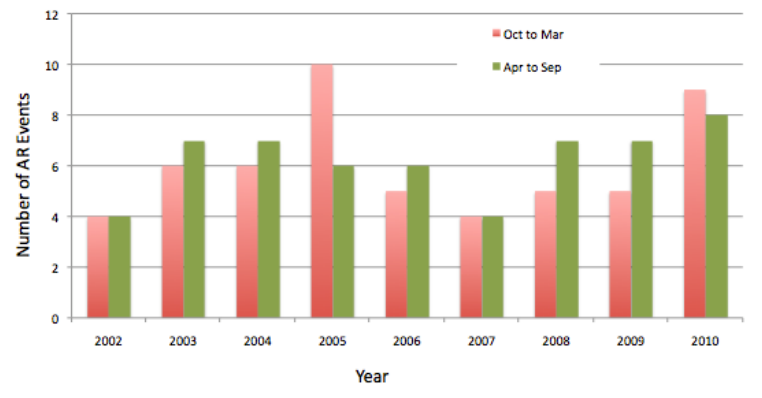

Figure 6: Yearly statistics of atmospheric events from observational data from http://www.remss.com/amsre/ 


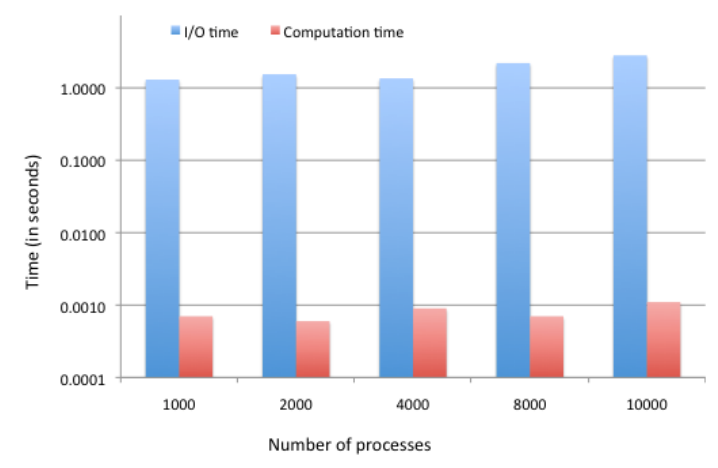

Figure 7: Weak scaling times

number of processes increases. While these costs stayed relatively constant, we noticed a small increase in the observed time. We attribute this to a small fraction of MPI processes taking longer than others to finish their processing. As the number of processes increase from 1000 to 10000 processes, the observed computation time fluctuates between $0.7 \mathrm{~ms}$ and $1.1 \mathrm{~ms}$, we believe that these fluctuations are random in nature (and not systematic).

The I/O time also increases slightly; the main reason for this increase is due to shared access to the same input file for reading filenames. As the number of processes increase, the time to read the file names increase from $0.29 \mathrm{~s}$ for 1000 processes to $1.54 \mathrm{~s}$ for 10,000 processes. In the same tests, the time to read the integrated water vapor data remains about the same, $1.01 \mathrm{~s} 1000$ processes and 1.32s for 10,000 processes.

In Figure 8, we show the aggregate I/O throughput against the number of processes. We calculated the aggregate $\mathrm{I} / \mathrm{O}$ throughput as the sum of $\mathrm{I} / \mathrm{O}$ throughput at each process. Since each process runs independently without any synchronization, measuring global I/O bandwidth for the application does not reflect I/O performance of the tool. As the number of processes increases, the $\mathrm{I} / \mathrm{O}$ throughput also increases. In the model dataset, each file contains five days of data and is therefore shared by five processes. Since each read request fetches a full stripe of data (1 MB in Lustre file system configured on Hopper), this 1 MB stripe of data includes all water vapor data for all five days. This explains achieving good I/O performance even though each process is expected to read only about $128 \mathrm{~KB}$ of data.

\subsection{Strong Scaling}

Figure 9 shows strong scaling results with a fixed data size related to 10,000 days. This experiment shows how the application scales as the number of processes increase, while the data size is fixed. The two bars show the $\mathrm{I} / \mathrm{O}$ time and the computation time. The sum of these two costs is equal to the total execution time of the algorithm. The upper trend line (dashed) refers to the I/O time if ideal speedup were achieved and the lower trend line represents computation time if ideal speedup were achieved. We calculated the time with ideal speedup in reference to the measured time when 100 processes were used. For

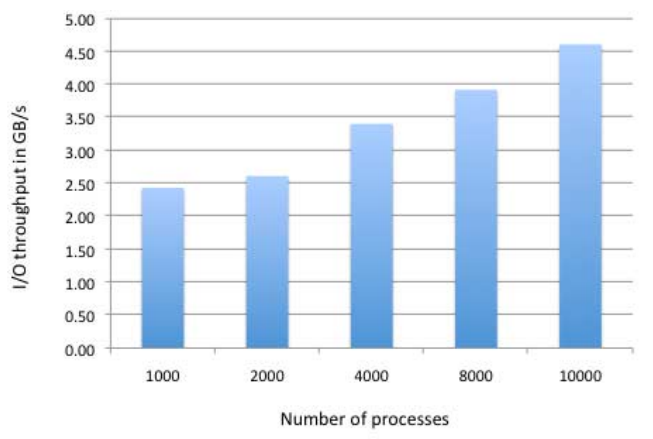

Figure 8: I/O performance with weak scaling

instance, if the $\mathrm{I} / \mathrm{O}$ overhead with 100 processes is $t$, then the I/O overhead with 200 processes is $t / 2$ and that with 500 processes is $t / 5$, and so on. The combination of reading file names from input file and the reading vapor data from climate data set dominate the overall execution time. The total I/O time constitutes $\sim 99 \%$ of the execution time.

In Figure 9, we see that the computation time speedup generally agrees with ideal scaling. This suggests that the computations are relatively load-balanced and amenable to parallelization. In this case, each process handles data from a number of different days, which minimizes the effect of random fluctuations discussed earlier.

The I/O times are very close to ideal speedup for the test cases with 100, 200 and 500 processes. As indicated before, five processes read from a single data file and their read operations are most likely served by a single disk read, which means that 100 OSTs can serve 500 processes. In going from 100 to 500 processes, our program is effectively using more OSTs from the file system; therefore the I/O time scales well. As more processes are used, it is no longer possible to have each OST serve five processes. This creates I/O contention and increases the time needed to complete the $\mathrm{I} / \mathrm{O}$ operations. We see that the $\mathrm{I} / \mathrm{O}$ time in Figure 9 goes above the expected value for ideal speedup when more than 1000 processes are used.

In Figure 10, we show the aggregate $\mathrm{I} / \mathrm{O}$ throughput as the number of processes increases with fixed data size. The I/O throughput increases as the number of processes increase up to 500 processes following the linear trend. From the 1000 processes case, the $\mathrm{I} / \mathrm{O}$ throughput falls short of ideal

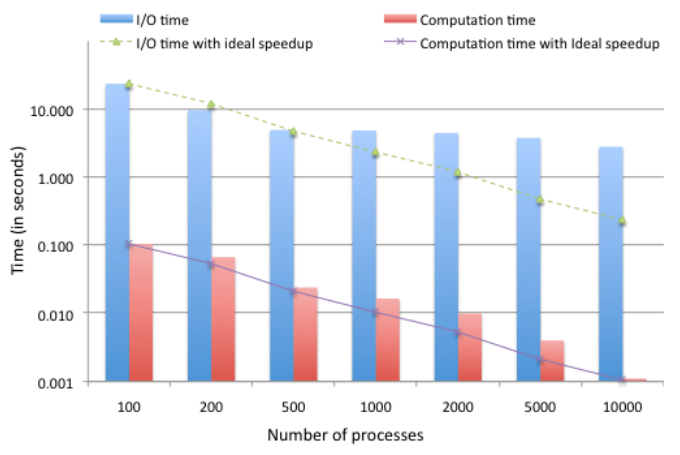

Figure 9: Strong scaling times 


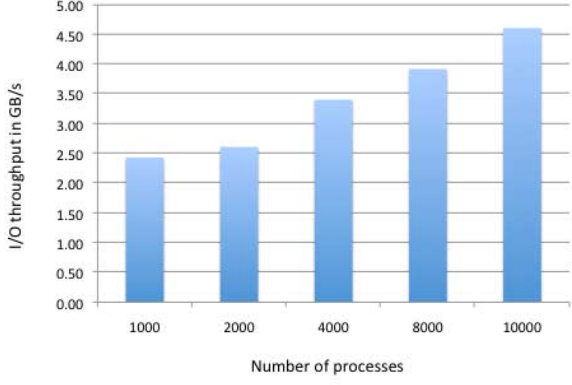

Figure 10: I/O Performance with strong scaling

growth. Nevertheless, the aggregate throughput still increases, reaching $4.6 \mathrm{~GB} / \mathrm{s}$ with 10,000 processes.

Our results indicate that our tool is quite well suited for weak scaling. Our tool can analyze more data with a larger number of processes. This will be useful in processing output data from century scale climate model integrations.

\section{CONCLUSIONS}

Atmospheric rivers are a type of rare weather event capable of transporting large amounts of water from tropical region to elsewhere. They are a important source of fresh water as well as a cause of severe flooding and wind damage. In this work, we have developed an efficient detection tool for automatically detecting ARs. We use a combination of thresholding, connected component labeling and verification steps to check for the presence of ARs. Our implementation was able to successfully detect $92 \%$ of ARs that make landfall; the results were verified against a manually curated results published by Dettinger, et al. We demonstrated good weak and strong scaling for our implementation. We applied our tool to a large 1TB dataset on 10,000 cores, and completed the processing in 3 seconds. This fully automated and highly parallelizable tool will enable climate scientists to effectively tackle large data challenges from next generation climate simulation output.

\section{ACKNOWLEDGMENTS}

This work was performed under the auspices of the U.S. Department of Energy (DOE) by the Lawrence Berkeley National Laboratory (LBNL) under contract DE-AC0376SF00098 (LBNL) and with support from the Office of Science (BER), U. S. Department of Energy. This research used resources of the National Energy Research Scientific Computing Center, which is supported by the Office of Science of the U.S. Department of Energy under Contract No. DE-AC02-05CH11231.

\section{REFERENCES}

[1] M. D. Dettinger, F. M. Ralph, T. Das, P. J. Neiman, and D. R. Cayan, "Atmospheric rivers, floods and the water resources of California", Water, 3(2):445-478, 2011.

[2] L. di Stefano and A. Bulgarelli, "A simple and efficient connected components labeling algorithm", In ICIAP '99: Proceedings of the 10th International Conference on Image
Analysis and Processing, page 322, Washington, DC, USA, 1999. IEEE Computer Society.

[3] M. B. Dillencourt, H. Samet, and M. Tamminen, "A general approach to connected-component labeling for arbitrary image representations", J. ACM, 39(2):253-280, 1992.

[4] H. Flatt, S. Blume, S. Hesselbarth, T. Schunemann, and P. Pirsch, "A parallel hardware architecture for connected component labeling based on fast label merging", In ASAP, pages 144-149. IEEE Computer Society, 2008.

[5] J. Greiner, "A comparison of parallel algorithms for connected components", In SPAA '94:pages 16-25, New York, USA, 1994.

[6] C.-Y. Lin, S.-Y. Li, and T.-H. Tsai, "A scalable parallel hardware architecture for connected component labeling", In ICIP, pages 3753-3756. IEEE, 2010.

[7] P. J. Neiman, F. M. Ralph, G. A. Wick, Y.-H. Kuo, T.-K. Wee, Z. Ma, G. H. Taylor, and M. D. Dettinger, "Diagnosis of an intense atmospheric river impacting the pacific northwest: Storm summary and offshore vertical structure observed with COSMIC satellite retrievals", Monthly Weather Review, 136(11):4398-4420, 2008.

[8] P. J. Neiman, A. B. White, F. M. Ralph, D. J. Gottas, and S. I. Gutman, "A water vapour flux tool for precipitation forecasting", Proceedings of Institution of Civil Engineers Water Management, 162:83-94, 2009.

[9] R. E. Newell, N. E. Newell, Y. Zhu, and C. Scott, "Tropospheric rivers? - A pilot study", Geophysical Research Letters, 19(24):2401-2404, 1992.

[10] F. M. Ralph, P. J. Neiman, G. N. Kiladis, K. Weickmann, and D. W. Reynolds, "A multiscale observational case study of a pacific atmospheric river exhibiting tropicalextratropical connections and a mesoscale frontal wave," Monthly Weather Review, 139(4):1169-1189, 2011.

[11] F. M. Ralph, P. J. Neiman, and G. A. Wick, "Satellite and CALJET aircraft observations of atmospheric rivers over the eastern north pacific ocean during the winter of 1997/98", Monthly Weather Review, 132(7):1721-1745, 2004.

[12] K.-B.Wang, T.-L. Chia, Z. Chen, and D.-C. Lou, "Parallel execution of a connected component labeling operation on a linear array architecture", Journal of Information Science And Engineering, 19:353-370, 2003.

[13] M. F. Wehner, G. Bala, P. Duffy, A. A. Mirin, and R. Romano, "Towards direct simulation of future tropical cyclone statistics in a high-resolution global atmospheric model”, In Advances in Meteorology, page 915303, 2010.

[14] A. B. White, F. M. Ralph, P. J. Neiman, D. J. Gottas, and S. I. Gutman, "The NOAA coastal atmospheric river observatory", In 34th Conference on Radar Meteorology.

[15] K. Wu, E. Otoo, and K. Suzuki, "Optimizing two-pass connected component labeling algorithms", Pattern Analysis \& Applications, 12(2):117-135, 2009.

[16] Y. Zhu and R. E. Newell, "Atmospheric rivers and bombs", Geophysical Research Letters, 21(18):1999-2002, 1994.

[17] Y. Zhu and R. E. Newell, "A proposed algorithm for moisture fluxes from atmospheric rivers", Monthly Weather Review - USA, 126(3):725-735, 1998. 